\title{
ARTICLE Establishment of a mouse model of cancer cachexia with spleen deficiency syndrome and the effects of atractylenolide I
}

\author{
Wan-li Zhang ${ }^{1,2}, \mathrm{Na} \mathrm{Li}^{3}$, Qiang Shen ${ }^{1}$, Men Fan ${ }^{2}$, Xiao-dong Guo ${ }^{4}$, Xiong-wen Zhang ${ }^{2}$, Zhou Zhang ${ }^{3}$ and Xuan Liu ${ }^{1}$
}

\begin{abstract}
Cancer cachexia is a multifactorial metabolic syndrome that affects $\sim 50 \%-80 \%$ of cancer patients, and no effective therapy for cancer cachexia is presently available. In traditional Chinese medicine, a large portion of patients with cancer cachexia was diagnosed as spleen deficiency syndrome and treated with tonifying TCMs that produce clinic benefits. In this study we established a new animal model of spleen deficiency and cancer cachexia in mice and evaluated the therapeutic effects of atractylenolide I, an active component of tonifying TCM BaiZhu, in the mouse model. Cancer cachexia was induced in male BALB/c mice by inoculation of mouse C26 colon adenocarcinoma cells, whereas spleen deficiency syndrome was induced by treating the mice with spleen deficiency-inducing factors, including limited feeding, fatigue, and purging. The mouse model was characterized by both cachexia and spleen deficiency characteristics, including significant body weight loss, cancer growth, muscle atrophy, fat lipolysis, spleen, and thymus atrophy as compared with healthy control mice, cancer cachexia mice, and spleen deficiency mice. Oral administration of atractylenolide I ( $20 \mathrm{mg} \cdot \mathrm{kg}^{-1}$ per day, for 30 days) significantly ameliorated the reduction in body weight and atrophy of muscle, fat, spleen, and thymus in mice with spleen deficiency and cachexia. The established model of spleen deficiency and cancer cachexia might be useful in the future for screening possible anticachexia TCMs and clarifying their mechanisms.
\end{abstract}

Keywords: cancer cachexia; traditional Chinese medicine; spleen deficiency; animal model; muscle atrophy; fat lipolysis; atractylenolide I; metabolic syndrome

Acta Pharmacologica Sinica (2020) 41:237-248; https://doi.org/10.1038/s41401-019-0275-z

\section{INTRODUCTION}

Cancer cachexia is a multifactorial metabolic syndrome that affects $\sim 50 \%-80 \%$ of cancer patients, depending on the tumor type [1]. In 2011, an international consensus statement defined cancer cachexia as "a multifactorial syndrome characterized by ongoing loss of skeletal muscle mass (with or without loss of fat mass) that cannot be fully reversed by conventional nutritional support and leads to progressive functional impairment" [2]. The primary clinical presentation of cancer cachexia is $>5 \%$ unintentional weight loss over 6 months, body mass index $<20 \mathrm{~kg} / \mathrm{m}^{2}$ and $>2 \%$ weight loss or an appendicular skeletal muscle index consistent with sarcopenia and $>2 \%$ weight loss [2]. Cancer cachexia negatively affects the quality of life, responsiveness to chemotherapy, and survival in advanced cancer patients [3]. Unfortunately, the etiopathogenesis of cancer cachexia is still not fully understood, although several factors and biological pathways, such as the production of cytokines and catabolic factors, abnormal metabolism, inflammation, insulin resistance, and negative protein/energy balance, have been reported to be involved [1, 4]. To date, no effective medical intervention has completely reversed cachexia, and there are no approved drug therapies for cancer cachexia [5].
Commonly used nutritional and anti-inflammatory strategies alone have proven ineffective for the management of symptoms of cancer cachexia [6]. Therefore, it is necessary and important to further clarify the etiopathogenesis of cancer cachexia and search for more options and candidates for cancer cachexia therapy.

The study of cancer cachexia is highly dependent on the use of suitable cancer cachexia animal models. A number of animal models of cancer cachexia have been developed [7]. Generally, in most animal models, tumor cells are implanted subcutaneously into experimental animals (mice or rats) and allowed to grow until the tumor burden induces cachexia symptoms [8]. The tumor cells used to inoculate animals include mouse colon adenocarcinoma C26 cells [9], mouse Lewis lung carcinoma (LLC) cells [10], human MCF-7 breast adenocarcinoma cells [11], human ES-2 OC cells [12], human gastric cancer-derived 85As2 cells [13], and Yoshida $\mathrm{AH}-$ 130 hepatoma cells [14]. Genetically engineered mouse models such as adenomatous polyposis coli (Apc) Min/ + mice, inhibin asubunit knockout mice, and Pdx1-cre;LSL-KrasG12D;INK4a/arffl/fl mice have also been used [15]. These animal models have contributed greatly to the study of cancer cachexia. However, no one model is ideal for mimicking the biological nuances of

\footnotetext{
${ }^{1}$ Institute of Interdisciplinary Integrative Biomedical Research, Shanghai University of Traditional Chinese Medicine, Shanghai 201203, China; ${ }^{2}$ Shanghai Engineering Research Center of Molecular Therapeutics and New Drug Development, School of Chemistry and Molecular Engineering, East China Normal University, Shanghai 200062, China; ${ }^{3}$ Shanghai

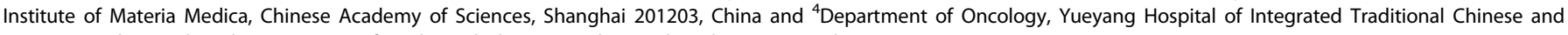
Western Medicine, Shanghai University of Traditional Chinese Medicine, Shanghai 200437, China

Correspondence: Xiong-wen Zhang (xwzhang@sat.ecnu.edu.cn) or Zhou Zhang (zzhang@simm.ac.cn) or Xuan Liu (xuanliu@shutcm.edu.cn)

These authors contributed equally: Wan-li Zhang, Na Li.
}

Received: 13 February 2019 Accepted: 17 June 2019

Published online: 24 July 2019 
patients and all their complexities, including tumor characteristics, host-tumor interactions, therapies, psychological stress, and comorbidities.

The TCM (traditional Chinese medicine) therapy system, including "Bian Zheng Lun Zhi", a treatment based on syndrome differentiation, is an essential part of the healthcare system in China. A TCM syndrome, in essence, is a characteristic profile of all clinical manifestations in one patient, and it helps to guide the design of individualized treatments [16]. Interestingly, most of the patients with cancer cachexia in China have been diagnosed with spleen deficiency syndrome [17-19]. In China, gastrointestinal cancers, including gastric cancer, liver cancer, esophageal cancer, and colorectal cancer, are major components of the national cancer burden [20]. Notably, in the development of gastrointestinal cancers, which exhibit a high cachexia incidence [21, 22], spleen deficiency syndrome is closely involved. In a clinical survey of 767 patients with gastric cancer, $32.86 \%$ of patients exhibited a syndrome of deficiency and cold in the spleen and stomach [23]. Spleen deficiency syndrome was also suggested to be pivotal to the pathogenesis of colorectal cancer development [24]. It is possible that cancer patients with spleen deficiency syndrome might be prone to developing cachexia, and the tumor burden might further exacerbate spleen deficiency syndrome. Therefore, an animal model combining cancer cachexia and spleen deficiency syndrome might be useful to mimic the clinical characteristics of cancer cachexia patients with spleen deficiency syndrome.

Among the presently available animal models of cancer cachexia, a model of mice bearing C26 mouse colon adenocarcinoma cells has been well accepted and widely used in both studies of the etiopathogenesis of cancer cachexia [25-28] and drug screening [29-32]. Additionally, mice with spleen deficiency induced by a combination of three kinds of inducing factors, i.e., limited feeding, fatigue, and purging, is also well accepted [3335]. Therefore, in the present study, we tried to establish a mouse model of spleen deficiency and cachexia by treating mice with both spleen deficiency-inducing factors (limited feeding, fatigue, and purging) to induce spleen deficiency syndrome and mouse C26 colon adenocarcinoma cells to induce cancer cachexia. At the same time, the spleen deficiency mouse model (mice treated with only spleen deficiency-inducing factors) and the cancer cachexia mouse model (mice subjected only to the inoculation of C26 cells) were also induced and compared with the spleen deficiency and cachexia mouse model. Furthermore, the potential benefits of traditional medicines and natural compounds in the treatment of cancer cachexia have attracted the interest of researchers [36-40]. Tonifying TCMs with the potency of "invigorating spleen and replenishing qi" have exhibited clinical efficacy in cancer cachexia therapy [41-43]. In the present study, the effects of atractylenolide I, a bioactive component of the tonifying TCM BaiZhu, on combined spleen deficiency and cachexia were observed to investigate the possible use of the mouse model of spleen deficiency and cachexia in drug screening of traditional medicines and natural herbs for cancer cachexia therapy.

\section{MATERIALS AND METHODS}

Chemicals and materials

Atractylenolide I (CAS No. 73069-13-3) with purity $>98 \%$ was purchased from Nature-Standard Co. Ltd. (Shanghai, China). Folium Sennae was purchased from Shanghai YuanYe Biotechnology Co. Ltd (Shanghai, China). To prepare the water extract of Folium Sennae, dried plant material was cut using an electric shredder, and $100 \mathrm{~g}$ of powder was extracted twice with $1 \mathrm{~L}$ of distilled water for $30 \mathrm{~min}$ each time. The combined aqueous extract solution was filtered and concentrated in a rotary evaporator to $100 \mathrm{~mL}$ (equal to $1 \mathrm{~g}$ of Folium Sennae in $1 \mathrm{~mL}$ of extraction) and stored at $-20^{\circ} \mathrm{C}$ before use. Other chemicals, except where specially noted, were purchased from Sigma-Aldrich Chemical Co. (St. Louis, MO, USA).

\section{Cell culture}

The C26 mouse colon adenocarcinoma cell line was obtained from the Shanghai Institute of Materia Medica, Chinese Academy of Sciences. Cells were cultured in RPMI-1640 medium (HyClone, Los Angeles, CA, USA) containing $10 \% \mathrm{FBS}$ at $37^{\circ} \mathrm{C}$ with $5 \% \mathrm{CO}_{2}$.

Animals and grouping

A total of 40 male BALB/c mice (6-8 weeks old) were purchased from Shanghai SLAC Laboratory Animal Co. Ltd. (Shanghai, China), and the animal care and experimental protocols were approved by the Institutional Animal Care and Use Committee of East China Normal University. The mice were maintained on a 12:12 h light-dark cycle in a temperature-controlled $\left(21-23^{\circ} \mathrm{C}\right)$ and specific pathogen-free room. All animals were acclimatized for 1 week before beginning the study. The animals were randomly grouped into five groups (eight mice in each group), i.e., the healthy control group (mice treated with natural saline for 30 days, i.g.), cachexia group (mice treated with natural saline for 30 days and at day 4 inoculated with C26 cells to induce cancer cachexia), spleen deficiency group (mice treated with spleen deficiencyinducing factors for 30 days), spleen deficiency and cachexia group (mice treated with spleen deficiency-inducing factors for 30 days and at day 4 inoculated with C26 cells to induce cancer cachexia), and spleen deficiency and cachexia + atractylenolide I group (spleen deficiency and cachexia mice treated with atractylenolide l, $20 \mathrm{mg} \cdot \mathrm{kg}^{-1}$ per day for 30 days, i.p.). During the course of the experiment, one animal in the spleen deficiency group was found dead at day 27, and one animal in the spleen deficiency and cachexia group was found dead at day 29. The results for these two animals were not included in the analysis.

\section{Induction of cancer cachexia in mice}

Cancer cachexia was induced in mice as reported in our previous study [44]. Briefly, $100 \mu \mathrm{L}$ of C26 colon adenocarcinoma cells $\left(1.0 \times 10^{6}\right.$ cells $)$ were implanted subcutaneously in the right flanks of the mice. Body weight, tumor volume, and food intake were measured every other day. The shortest diameter $(x)$ and longest diameter $(y)$ of the tumor were measured using calipers. Tumor volume was calculated following the formula: $V=x \times x \times y \times 0.5$.

\section{Induction of spleen deficiency in mice}

Spleen deficiency was induced in mice using three inducing factors, limited feeding, fatigue, and purging, similar to previous reports [33-35]. First, for limited feeding, food (standard rodent chow) was provided to the mice only from 8 a.m. to 8 p.m. each day, while water was available ad libitum. Second, to induce fatigue, the mice were forced to swim until they reached exhaustion once every day. Exhaustion of the mice was classified as the loss of coordinated movements and the inability to rise to the water surface within $10 \mathrm{~s}$. Third, to induce purging, the mice were treated with the water extract of Folium Sennae $(20 \mu \mathrm{L} / \mathrm{mg}$ body weight every day from days 1 to 16 and $28 \mu \mathrm{L} / \mathrm{mg}$ body weight every day from days 17 to 30 ).

Induction of spleen deficiency and cachexia and treatment with atractylenolide I

Spleen deficiency and cachexia were induced by first treating the mice with three spleen deficiency-inducing factors for 4 days and then inoculating $1.0 \times 10^{6} \mathrm{C} 26$ cells and letting the tumor grow for 26 days (treatment with spleen deficiency-inducing factors was continued during these 26 days). In the spleen deficiency and cachexia + atractylenolide I group, the mice were treated with atractylenolide I ( $20 \mathrm{mg} \cdot \mathrm{kg}^{-1}$ day, i.p.) for 30 days (from the beginning of the experiment to the end of the experiment) in addition to the induction of spleen deficiency and cachexia. 
Monitoring the physiological parameters of the mice During the course of the experiment, the body weight, food intake, and tumor volume of each mouse were measured every other day. At the end of the experiment, the mice were euthanized by $\mathrm{CO}_{2}$ inhalation. Tissues including tumor, gastrocnemius muscle, soleus muscle, epididymal white adipose tissue (eWAT), thymus, and spleen tissue were isolated and weighed. Furthermore, samples of the gastrocnemius muscle, eWAT, spleen, and thymus tissues were fixed in $4 \%$ paraformaldehyde for $24 \mathrm{~h}$. Paraffin-embedded tissues were then cut into $10 \mu \mathrm{m}$ sections and subjected to H\&E staining by standard procedures. The samples were observed under an Olympus IX70 fluorescence microscope. Ten fields from each image were selected randomly for calculation of myofiber cross-sectional area or cell diameter of adipocytes using ImageJ software.

Western blotting assay

Western blotting assay was performed as described previously [44]. Briefly, the tissues (gastrocnemius muscle or eWAT) were homogenized in RIPA buffer plus a phosphatase protease inhibitor by a tissue lyser $(50 \mathrm{HZ}, 15 \mathrm{~min})$ purchased from Qiagen. The lysates were centrifuged at $13000 \mathrm{r} / \mathrm{min}$ for $30 \mathrm{~min}$ at $4{ }^{\circ} \mathrm{C}$. The supernatant was quantified for protein concentration using the BCA Protein Assay Kit (Beyotime, Shanghai, China). Equal amounts of protein samples were separated by $10 \%$ SDS-PAGE gel electrophoresis and transferred to a polyvinylidene fluoride membrane. The polyvinylidene fluoride membranes were blocked in $5 \%$ nonfat milk in phosphate-buffered saline containing $0.1 \%$ Tween 20 (TPBS) for $1 \mathrm{~h}$ at room temperature and then incubated with primary antibodies diluted in $5 \%$ BSA-TPBS at $4{ }^{\circ} \mathrm{C}$ overnight. The primary antibodies used were as follows: mouse anti-MHC monoclonal antibody (1:1000, DSHB, lowa City, IA, USA), rabbit anti-MyoD polyclonal antibody (1:1000, Santa Cruz Biotechnology, Dallas, TX, USA), rabbit anti-Myogenin polyclonal antibody (1:1000, Cell Signaling Technology, Danvers, MA, USA), rabbit anti-MuRF-1 monoclonal antibody (1:1000, Abcam, Cambridge, MA, USA), rabbit anti-HSL polyclonal antibody $(1: 1000$, Cell Signaling Technology), rabbit anti-p-HSL polyclonal antibody (1:1000, Cell Signaling Technology), rabbit anti-Perilipin-1 polyclonal antibody (1:1000, Cell Signaling Technology), rabbit anti-UCP1 monoclonal antibody (1:1000, Cell Signaling Technology), and HRP-conjugated anti-actin antibody (1:1000, Santa Cruz Biotechnology). An HRPconjugated goat anti-mouse secondary antibody (1:5000, Multi Sciences, Hangzhou, China) and goat anti-rabbit secondary antibody (1:5000, Multi Sciences) were incubated with the membranes for $1 \mathrm{~h}$ in $5 \%$ nonfat milk in TPBS. An ECL Chemiluminescent Kit (Thermo Fisher, Waltham, MA, USA) was used to visualize the antibody-antigen interaction, and chemical luminescence of membranes was detected by Amersham Imager 600 (GE).

\section{Immunohistochemical assay}

Immunohistochemical assay was performed as described previously [45]. Briefly, spleen or thymus tissues from mice were embedded in a paraffin block and subjected to immunohistochemistry. After being deparaffinized and hydrated, the tissues were permeabilized with $0.5 \%$ Triton $X-100$ in PBS for 10 min and then hybridized with the rabbit anti-CD4 polyclonal antibody (1:200; GB13064-2; Servicebio, Wuhan, China) or the rabbit anti-CD8 polyclonal antibody (1:1000; GB11068; Servicebio). An HRP-conjugated goat anti-rabbit antibody (1:200; GB23303; Servicebio) was used as the secondary antibody. Images were captured with a microscope (Olympus IX73, Tokyo, Japan). Three images with the same background light were taken randomly in each group, and the view was filled with tissue when photographing. Image-Pro Plus software was used to analyze the integrated optical density of the positive signal and the area of the tissue. The average optical density was calculated with the formula (integrated optical density)/(area). A high average optical density represented a high level of positive signal.

Statistical analysis

Values were expressed as the mean \pm standard error of the mean (SEM). All statistical analyses were performed using GraphPad Prism 5.0 software, and comparisons between two groups were analyzed using two-tailed Student's $t$-test. A $P$ value $<0.05$ was considered statistically significant.

\section{RESULTS}

Body weight loss in different groups of mice

The profiles of time-related change in body weight and tumor-free body weight in different groups of mice are shown in Fig. 1a, b, respectively. First, as shown in Fig. $1 \mathrm{a}, \mathrm{b}$, the body weight and the tumor-free body weight of the cachexia group decreased gradually after inoculation of C26 tumor cells at day 4 . The results were in accordance with previous reports of C26 cancer cachexia model $[27,44]$ and confirmed the successful induction of cancerrelated cachexia in the mice. Second, the three groups with spleen deficiency syndrome (spleen deficiency group, spleen deficiency with cachexia group, and spleen deficiency with cachexia $+\mathrm{Al}$
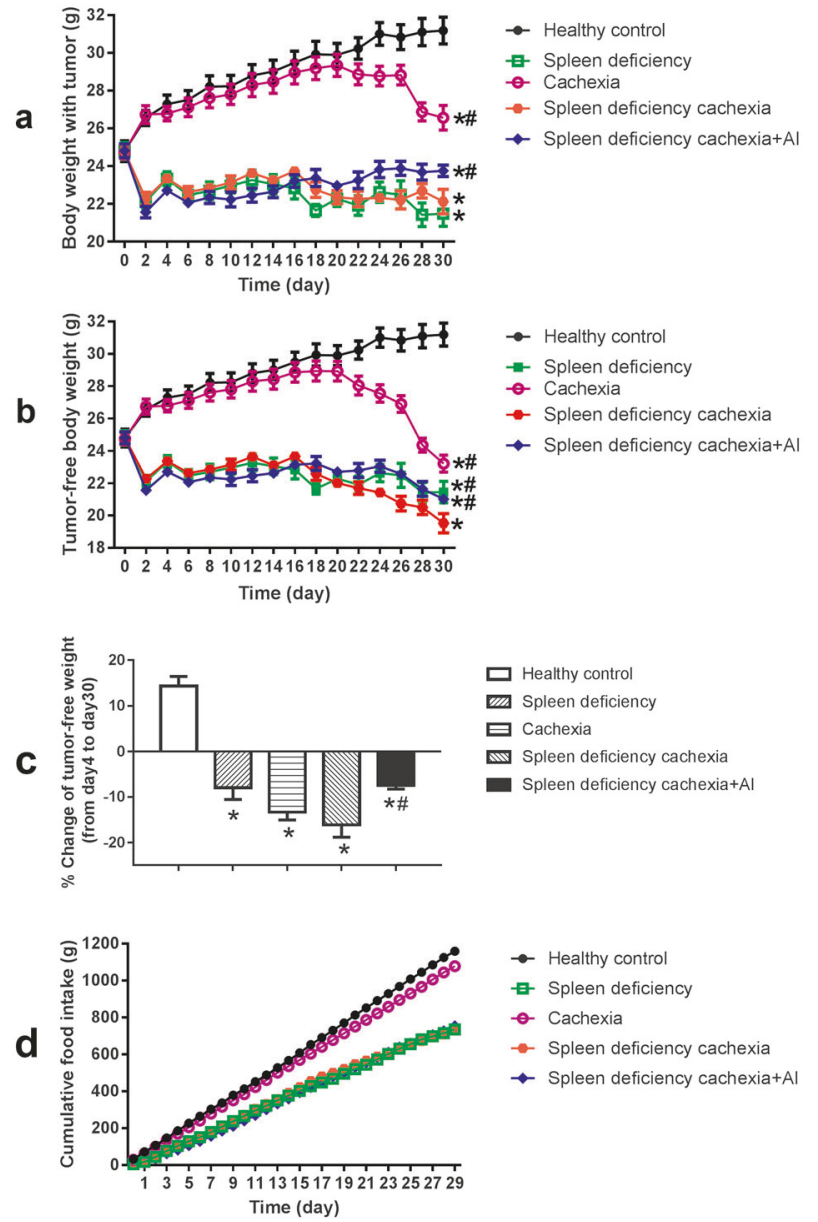

Fig. 1 Body weight and food intake of different groups of mice. a Profiles of the time-related change in the body weight of mice. b Profiles of the time-related change in the tumor-free body weight of mice. c Percentage of change in the tumor-free body weight of mice from day 4 to day 30. d Cumulative food intake of mice. The data presented are the mean \pm SEM. ${ }^{*} P<0.05$ versus healthy control group. ${ }^{\#} P<0.05$ versus spleen deficiency with cachexia group 
group) exhibited relatively low body weight. The low body weight in mice with spleen deficiency might result from treatment with spleen deficiency-inducing factors (limited feeding, fatigue, and purging). Inoculation of C26 tumor cells exacerbated the decrease in body weight of mice with spleen deficiency syndrome. At day 20 , the tumor-free body weight of mice with spleen deficiency and cachexia became lower than that of the spleen deficiency mice. At the end of the experiment (day 30), the difference between the tumor-free body weight of the spleen deficiency with cachexia group and that of spleen deficiency group was significant. Notably, treatment with atractylenolide I (Al) ameliorated the decrease in body weight of mice with spleen deficiency and cachexia; thus, the tumor-free body weight of the spleen deficiency with cachexia + Al group was significantly higher than that of the spleen deficiency with cachexia group at day 30. As shown in Fig. 1c, calculations of the percentage of change in tumor-free body weight from day 4 to day 30 also showed similar results. The significant difference between the spleen deficiency with cachexia + Al group and the spleen deficiency with cachexia group suggested the efficacy of atractylenolide I treatment in protecting mice with spleen deficiency and cachexia from an extreme loss of body weight. The cumulative food intake of the mice is shown in Fig. 1d. The inoculation of C26 tumors caused a decrease in the food intake of mice in the cachexia group compared with that of mice in the healthy control group. Because food limitation is involved in the induction of spleen deficiency, the food intake of mice in the spleen deficiency group and the spleen deficiency with cachexia group was much lower than that in the cachexia group. There was no difference in food intake between the spleen deficiency group and the spleen deficiency with cachexia group. Treatment with atractylenolide I did not change the food intake of the mice.

Tumor growth in different groups of mice

The profiles of the time-related change in tumor volume for different groups are shown in Fig. 2a. The photographs and weights of the tumor tissues isolated at the end of the experiment are shown in Fig. 2b, c, respectively. As shown in Fig. 2a-c, tumor growth was slightly decreased in the spleen deficiency with cachexia group compared with that in the cachexia group. However, the difference in tumor volume and tumor weight between the two groups was not significant. Atractylenolide I treatment could not inhibit the growth of tumors in mice with spleen deficiency and cachexia. These results indicated that the ameliorating effects of atractylenolide I on body weight loss in mice with spleen deficiency and cachexia might not result from inhibition of tumor growth.

Muscle atrophy in different groups of mice

The results of weight and histological analysis of muscle tissues in different groups are shown in Fig. 3. As shown in Fig. 3a-d, both inoculation of C26 tumors and induction of spleen deficiency could cause a decrease in muscle weight. The weights of muscle tissues, including the gastrocnemius muscle (Fig. 3a, b) and soleus muscle (Fig. 3c, d), in both the cachexia group and spleen deficiency group were significantly lower than those of the healthy control group. Notably, the weights of muscles in the spleen deficiency with cachexia group were even lower than those in the cachexia or spleen deficiency group. The difference in the weight of the gastrocnemius muscle between the spleen deficiency with cachexia group and the cachexia group was significant. These results suggested that muscle atrophy in the spleen deficiency with cachexia group was evident and even stronger than that in the cachexia group. Muscle atrophy caused a decrease in the myofiber area of muscle tissues. As shown in Fig. $3 e$ (representative results) and Fig. $3 f$ (quantitative results), analysis of H\&E-stained muscle sections also showed that the
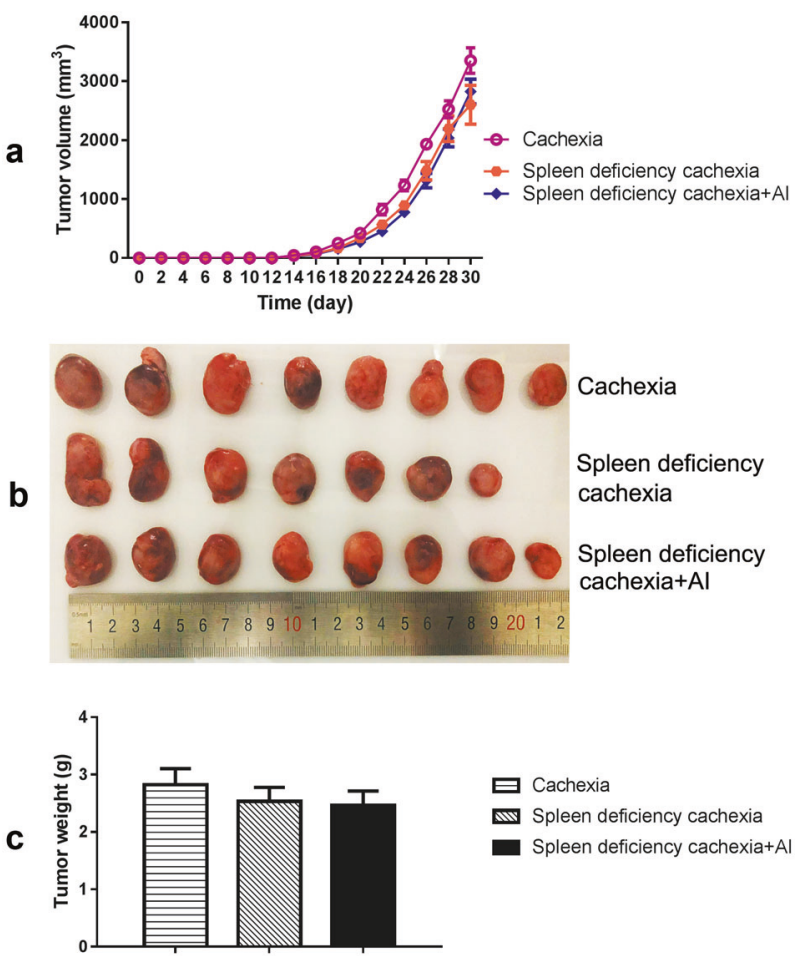

Fig. 2 Tumor growth in different groups of mice. a Time-related tumor growth curve in mice. b Photo of tumor tissues isolated from mice at the end of the experiment. c Weight of isolated tumor tissues. The data presented are the mean \pm SEM

myofiber area in the spleen deficiency with cachexia group was smaller than that of the cachexia group or spleen deficiency group. Atractylenolide I treatment could significantly ameliorate the decrease in muscle weight in mice with spleen deficiency and cachexia. The weight values of both gastrocnemius muscle (Fig. 3b) and soleus muscle (Fig. 3d) in the spleen deficiency with cachexia + Al group were significantly higher than those in the spleen deficiency with cachexia group. The decrease in the myofiber area of mice with spleen deficiency and cachexia was also ameliorated by treatment with atractylenolide I (Fig. 3e, f).

Fat lipolysis in different groups of mice

The results of weight and histological analysis of eWAT fat tissue in different groups are shown in Fig. 4. As shown in Fig. 4, the weights of eWAT in the cachexia group, spleen deficiency group, and spleen deficiency with cachexia group were all significantly lower than those of the healthy control group (Fig. 4a, b). The weight values of eWAT in the spleen deficiency with cachexia group were even significantly lower than those in the cachexia or spleen deficiency group. Histological analysis of H\&E-stained eWAT sections (representative results in Fig. $4 c$ and quantitative results in Fig. 4d) also showed that the diameter of adipocytes in the spleen deficiency with cachexia group was smaller than that in the cachexia group or spleen deficiency group. These results suggested that fat lipolysis in spleen deficiency with cachexia was evident and even stronger than that in the cachexia group or spleen deficiency group. Notably, atractylenolide I treatment could significantly ameliorate the decrease in fat weight in mice with spleen deficiency and cachexia. The weight values of eWAT in the spleen deficiency with cachexia + Al group were significantly higher than those in the spleen deficiency with cachexia group. The decrease in the diameter of adipocytes in mice with spleen deficiency and cachexia was also ameliorated by treatment with atractylenolide I (Fig. 4c, d). 
a

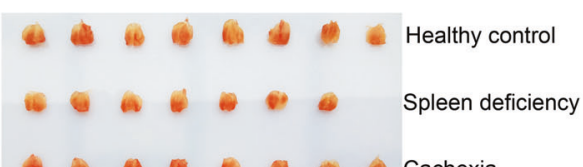

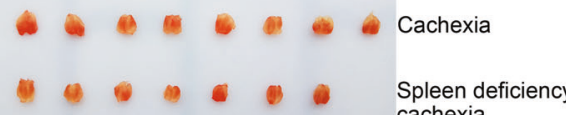

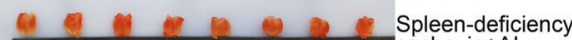
cachexia+Al

c

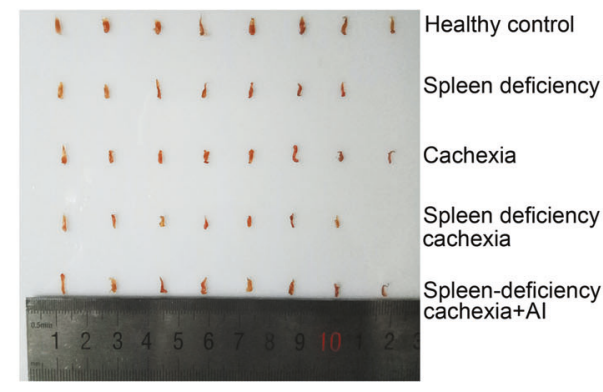

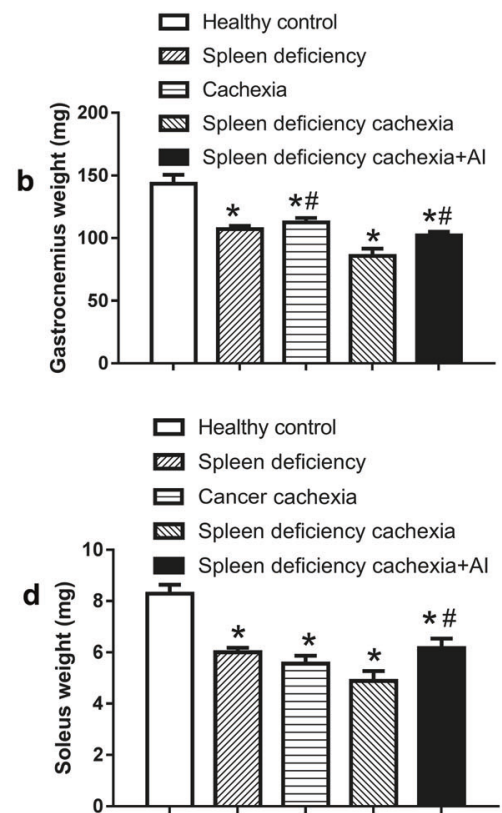

Cachexia e
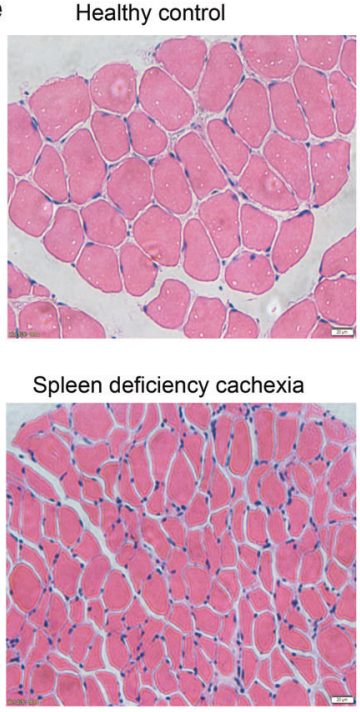

Spleen deficiency

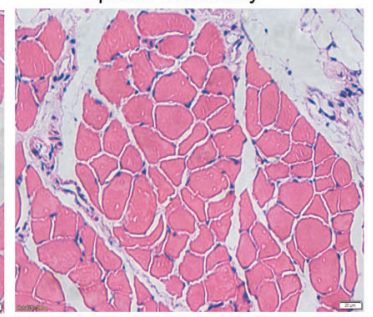

Spleen deficiency cachexia+Al

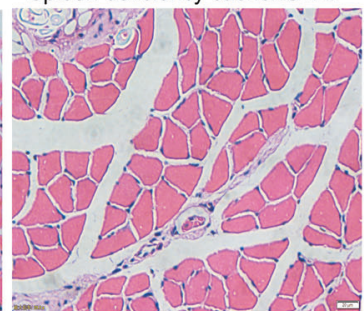

f

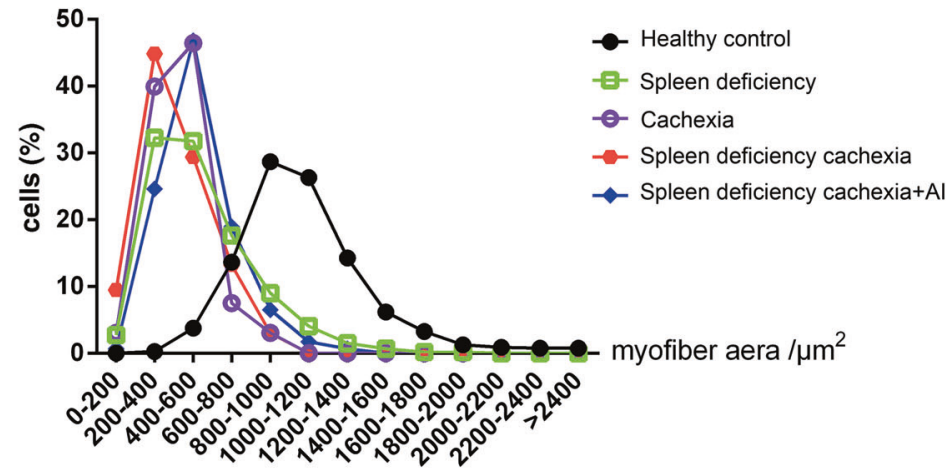

Fig. 3 Muscle atrophy in different groups of mice. a Photo of gastrocnemius muscle tissues. b Weight of gastrocnemius muscle tissues. c Photo of soleus muscle tissues. d Weight of soleus muscle tissues. e Representative photo of H\&E-stained sections of gastrocnemius muscle tissues. Bar $=20 \mu \mathrm{m}$. $\mathbf{f}$ Results of quantitative analysis of myofiber area in gastrocnemius muscle sections. The data presented are the mean \pm SEM. ${ }^{*} P<0.05$ versus healthy control group; ${ }^{\#} P<0.05$ versus spleen deficiency with cachexia group 

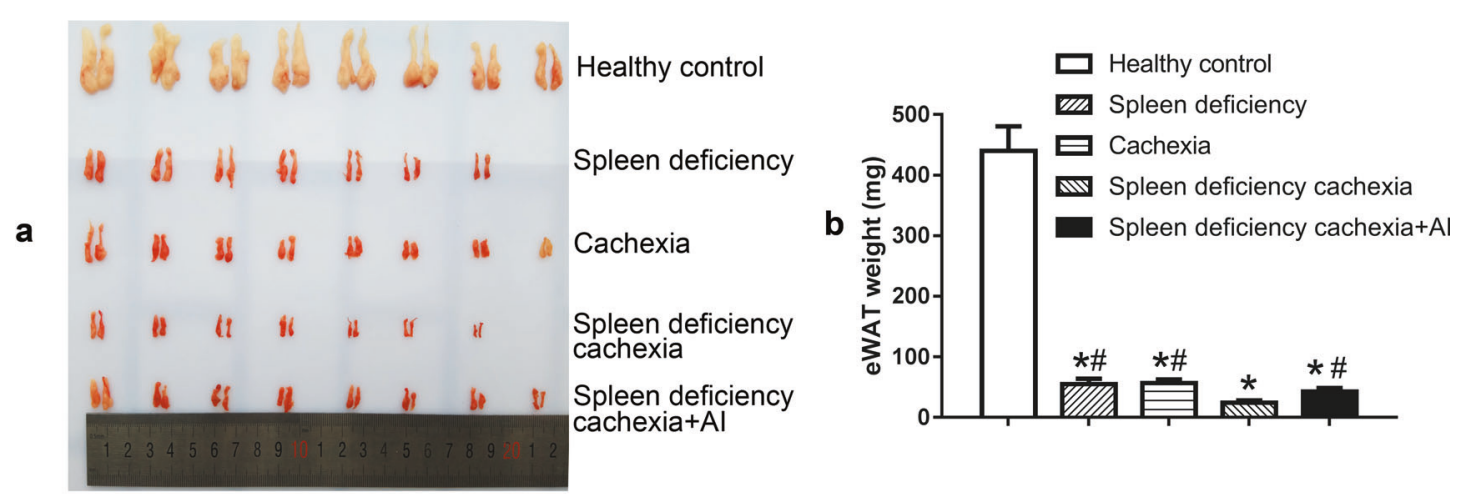

C
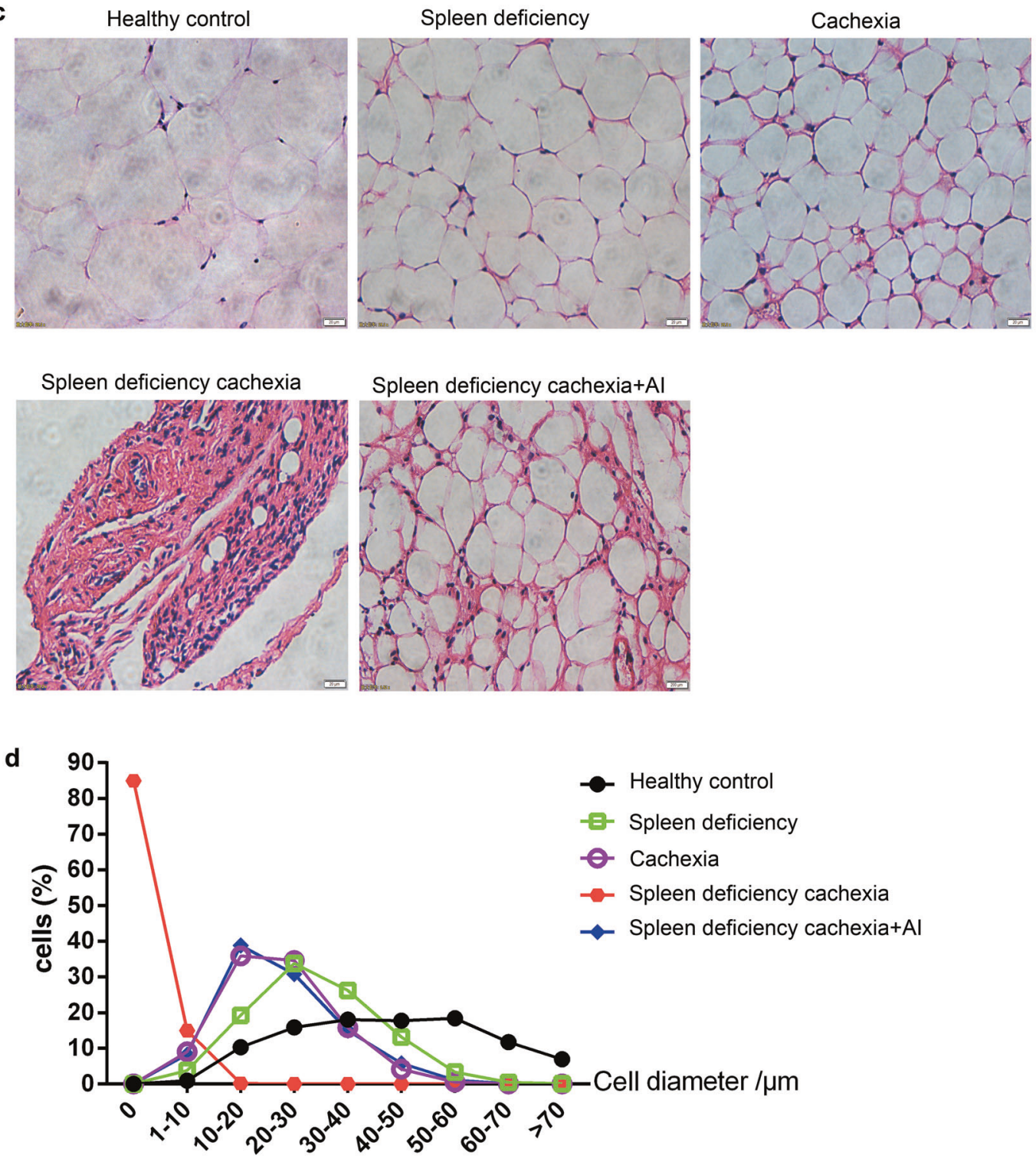

Fig. 4 Fat lipolysis in different groups of mice. a Photo of eWAT. b Weight of eWAT. c Representative photo of H\&E-stained sections of eWAT. Bar $=20 \mu \mathrm{m}$. d Results of quantitative analysis of the diameter of adipocytes in eWAT sections. The data presented are the mean \pm SEM. ${ }^{*} P<0.05$ versus healthy control group; ${ }^{\#} P<0.05$ versus spleen deficiency with cachexia group 

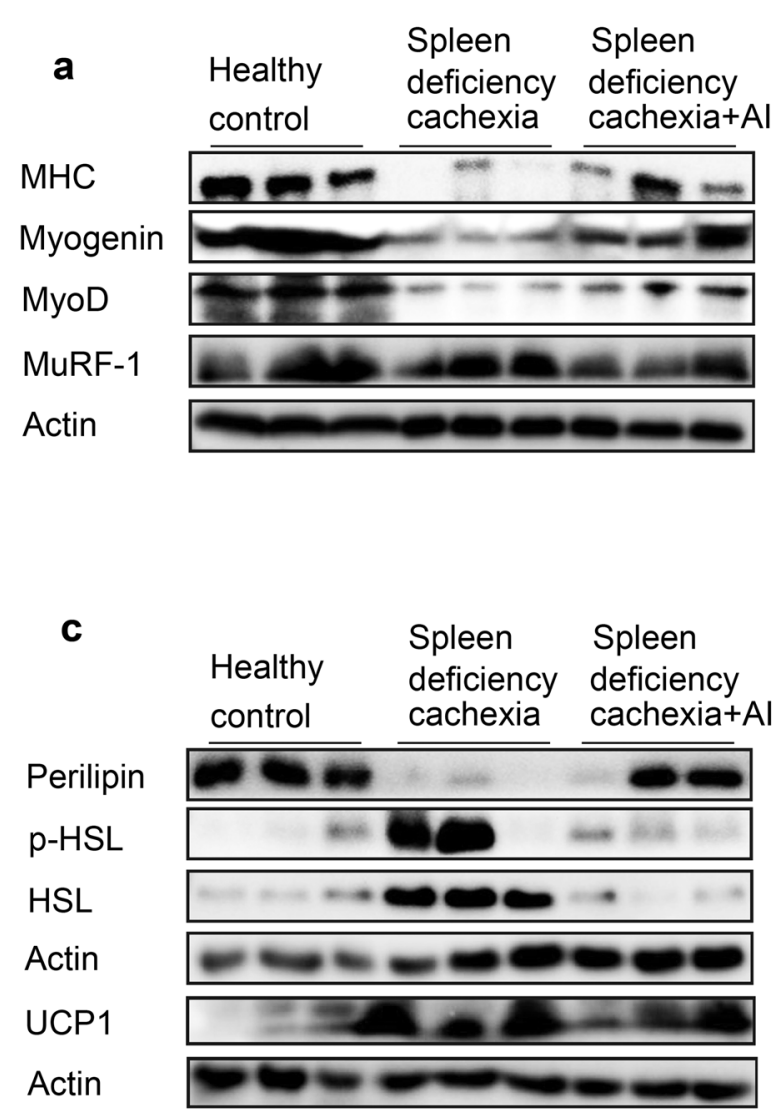

b

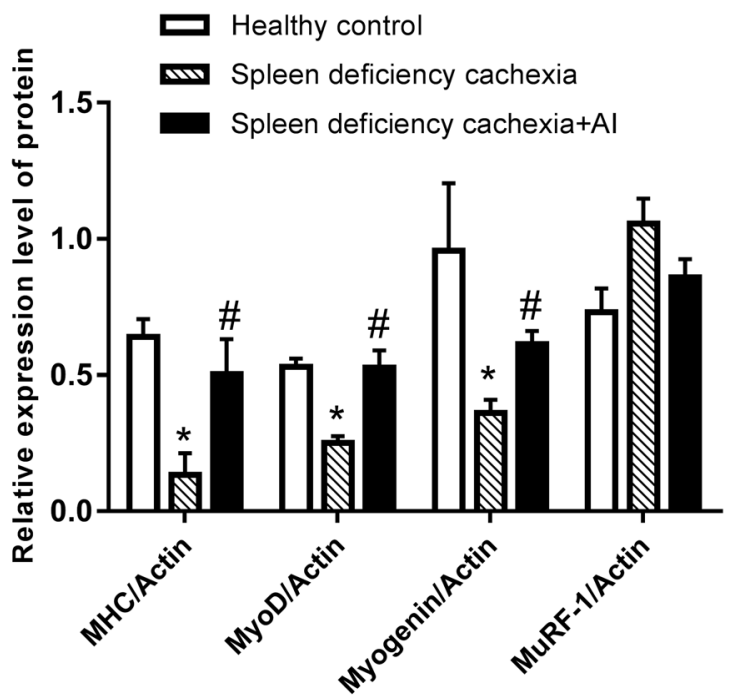

d

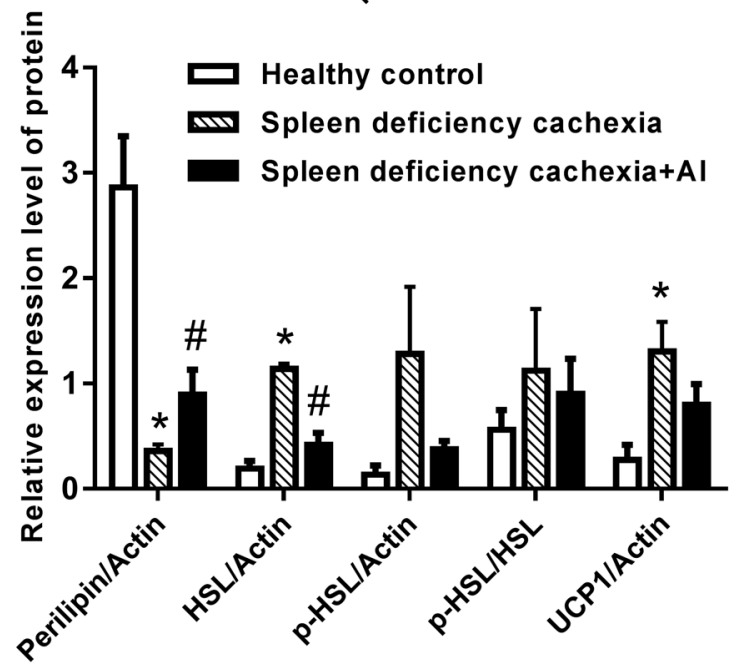

Fig. 5 Amelioration of muscle atrophy and fat lipolysis under atractylenolide I treatment. a Results of a Western blot assay for MHC, Myogenin, MyoD, and MuRF-1 in gastrocnemius muscle tissues. b Quantitative analysis of the results from a. c Results of a Western blot assay for Perilipin, $\mathrm{p}-\mathrm{HSL}, \mathrm{HSL}$, and UCP1 in eWAT. d Quantitative analysis of the results from c. Data presented are the mean $\pm \mathrm{SEM}$. ${ }^{*} P<0.05$ versus healthy control group; ${ }^{\#} P<0.05$ versus spleen deficiency with cachexia group

Amelioration of muscle atrophy and fat lipolysis under atractylenolide I treatment

The results of Western blot assays for proteins related to muscle atrophy or fat lipolysis in gastrocnemius muscle tissues and eWAT are shown in Fig. 5. As shown in Fig. 5a, b, treatment with atractylenolide I significantly ameliorated the decrease in the level of the myosin heavy chain (MHC) and inhibited the decrease in the myogenic regulatory factors Myogenin and MyoD in gastrocnemius muscle tissues from mice with spleen deficiency and cachexia. Atractylenolide I treatment also partly inhibited the increase in the protein degradation factor MuRF-1 in gastrocnemius muscle tissues. As shown in Fig. $5 c$, d, treatment with atractylenolide I significantly ameliorated the decrease in the level of the lipid droplet protein perilipin-1 in eWAT from mice with spleen deficiency and cachexia. Both the levels of HSL (hormone sensitive lipase) and p-HSL increased in mice with spleen deficiency and cachexia compared with those in the healthy control group. Atractylenolide I treatment significantly inhibited the increase in HSL and partly decreased the level of activated HSL ( $p-H S L)$ in eWAT. In eWAT from mice with spleen deficiency and cachexia, a significant increase in the level of uncoupling protein 1 (UCP1) was observed, and atractylenolide I treatment could partly inhibit this increase in UCP1.
Thymus and spleen weight in different groups of mice The weights of the thymus and spleen tissues in the different groups are shown in Fig. 6. As shown in Fig. 6a (thymus weight) and Fig. $6 \mathrm{~b}$ (thymus index, the ratio of thymus weight to body weight), the weight of the thymus decreased in the cachexia group, spleen deficiency group, and spleen deficiency with cachexia group compared with that in the healthy control group. The thymus index in the spleen-deficiency with cachexia group was even lower than that in the cachexia group or spleen deficiency group. Atractylenolide I treatment could significantly ameliorate the decrease in thymus weight in mice with spleen deficiency and cachexia. As to the change in spleen tissue (spleen weight in Fig. $6 \mathrm{c}$ and spleen index in Fig. $6 \mathrm{~d}$ ), the weight of the spleen decreased in the spleen deficiency group but increased in the cachexia group. In the spleen deficiency with cachexia group, the spleen weight was significantly lower than that in the healthy control group, while the spleen index was similar to that in the healthy control group. Atractylenolide I treatment could increase the weight of the spleen; thus, both the spleen weight and the spleen index in the spleen deficiency with cachexia + Al group were significantly higher than those in the spleen deficiency with cachexia group. 

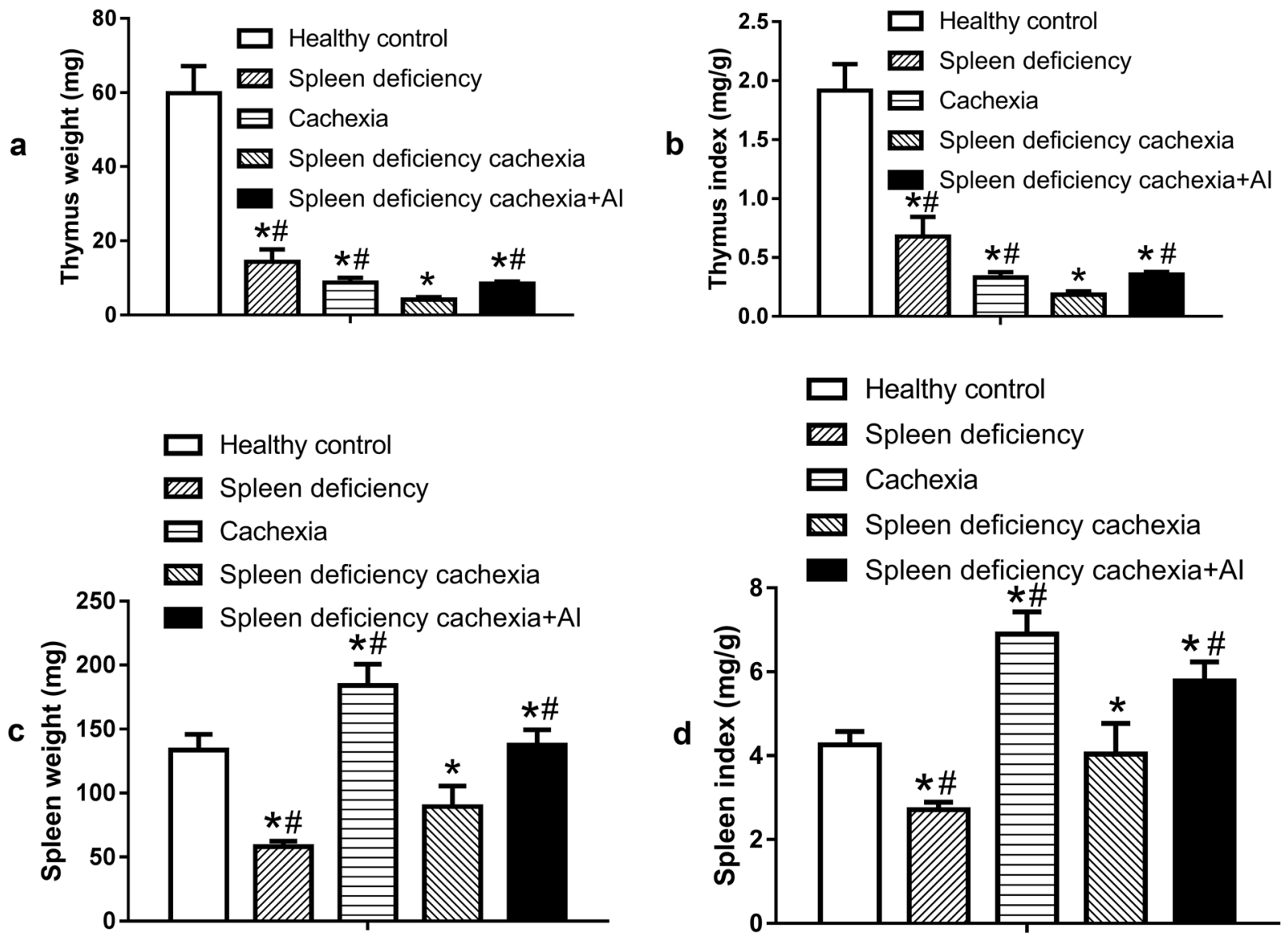

Fig. 6 Atrophy of the thymus and spleen in different groups of mice. a Weight of the thymus. $\mathbf{b}$ Thymus index (ratio of thymus weight to body weight). c Weight of the spleen. d Spleen index (ratio of spleen weight to body weight). The data presented are the mean \pm SEM. ${ }^{*} P<0.05$ versus healthy control group; ${ }^{\#} P<0.05$ versus spleen deficiency with cachexia group

Amelioration of thymus and spleen atrophy under atractylenolide I treatment

The results of histological analysis of the spleen and thymus tissues with or without atractylenolide I treatment are shown in Fig. 7a. As shown in Fig. 7a, in healthy control mice, the spleen tissue exhibited clearly identifiable regions of red and white pulp, while the thymus tissue exhibited distinct areas of cortex and medulla. In contrast, the typical textures of the spleen and thymus were largely destroyed in mice with spleen deficiency and cachexia. Atractylenolide I treatment attenuated the progression of atrophy and partly inhibited the destruction of the architecture of the spleen and thymus tissues in mice with spleen deficiency and cachexia. Furthermore, as shown in Fig. 7b, c, in the spleens of mice with spleen deficiency and cachexia, the number of $\mathrm{CD}^{+}$ cells significantly decreased, while the number of $\mathrm{CD}^{+}$cells increased. Atractylenolide I treatment significantly inhibited both the decrease in the number of $\mathrm{CD}^{+}$cells and the increase in the number of $\mathrm{CD}^{+}$cells. Similar results were observed in the analysis of the thymus tissues. As shown in Fig. 7d, e, atractylenolide I treatment also significantly inhibited the decrease in the number of $\mathrm{CD} 4^{+}$cells and the increase in the number of $\mathrm{CD}^{+}$cells in thymus tissues.

\section{DISCUSSION}

In the present study, an animal model of cancer cachexia with spleen deficiency syndrome was established by combining induction of spleen deficiency and induction of cancer cachexia in mice. Previous reports correlated the molecular profile of spleen deficiency syndrome with immunological dysfunction as well as abnormal energy metabolism [46]. Therefore, spleen deficiency syndrome is frequently observed in cancer patients, especially those suffering from cancer cachexia [17-19]. The mice with spleen deficiency and cachexia established in the present study exhibited both characteristics of cancer cachexia and characteristics of spleen deficiency.

The mice with spleen deficiency and cachexia exhibited the characteristics of cancer cachexia, i.e., weight loss, muscle atrophy, and lipid lipolysis. Although the induction of spleen deficiency in mice did not significantly affect the growth of tumors, it exacerbated weight loss, muscle atrophy, and lipid lipolysis caused by cancer cachexia. The mice with spleen deficiency and cachexia decreased their initial body weight by $\sim 20 \%$ after 30 days. Spleen deficiency alone or cancer cachexia alone also caused a decrease in the body weight of mice. Notably, the tumorfree body weight of mice with spleen deficiency and cachexia was significantly lower than that of cancer cachexia mice or spleen deficiency mice. As shown by the results of measuring the muscle weight and fat weight of the mice, both a decrease in muscle weight and a decrease in fat weight contribute to the loss of body weight in mice with spleen deficiency and cachexia. The myofiber area of mice with spleen deficiency and cachexia was smaller than that of cancer cachexia mice or spleen deficiency mice. Similarly, the diameter of adipocytes in mice with spleen deficiency and cachexia was also smaller than that of cancer cachexia mice or spleen deficiency mice. Overall, mice with spleen deficiency and cachexia exhibited strong muscle atrophy and lipid lipolysis, which are typical cachexia characteristics.

The mice with spleen deficiency and cachexia in the present study also exhibited the characteristics of spleen deficiency, i.e., 

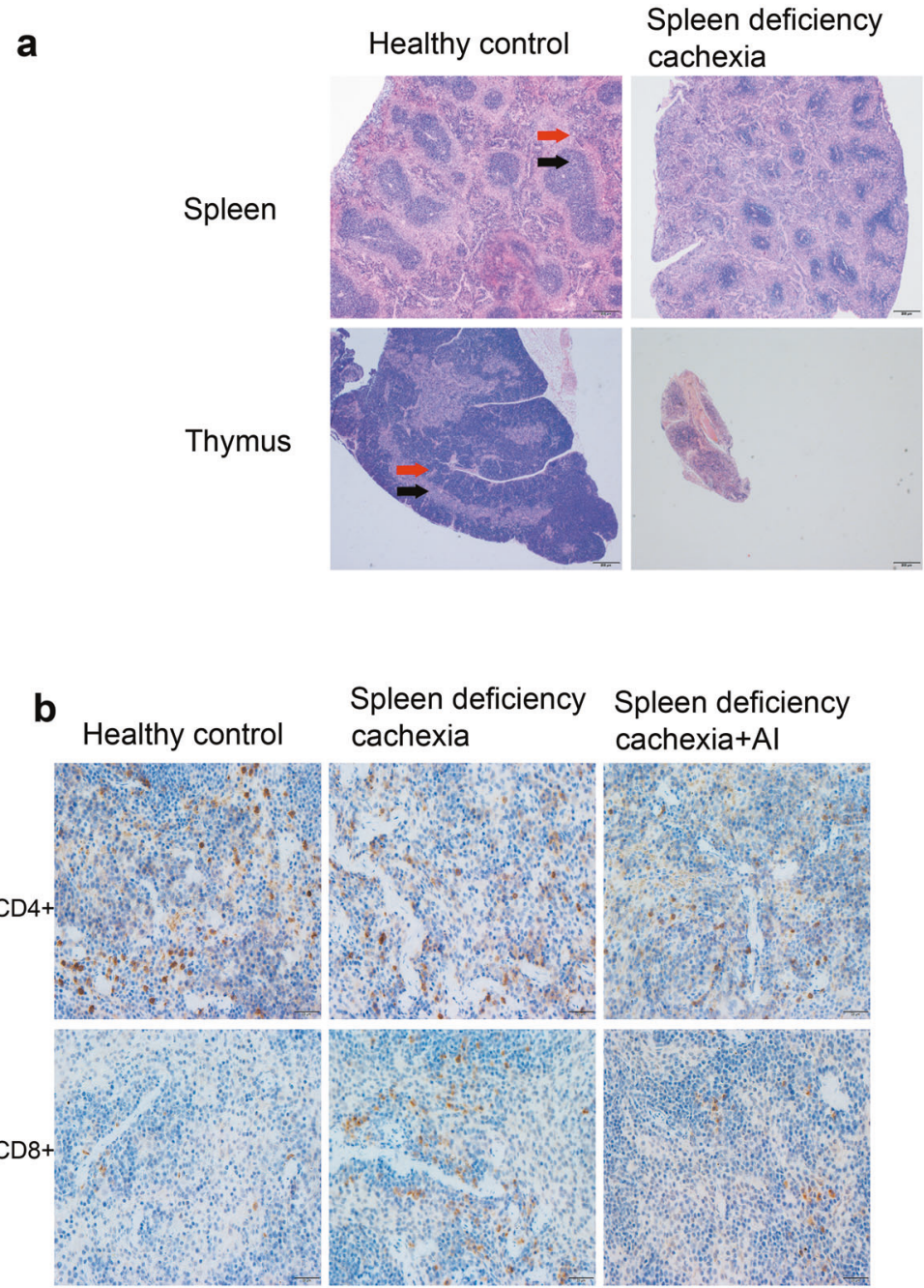

Spleen deficiency cachexia

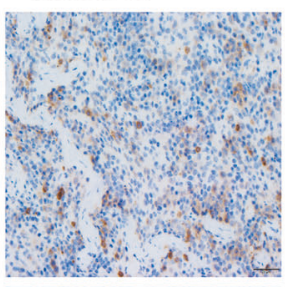

\section{cachexia+Al}
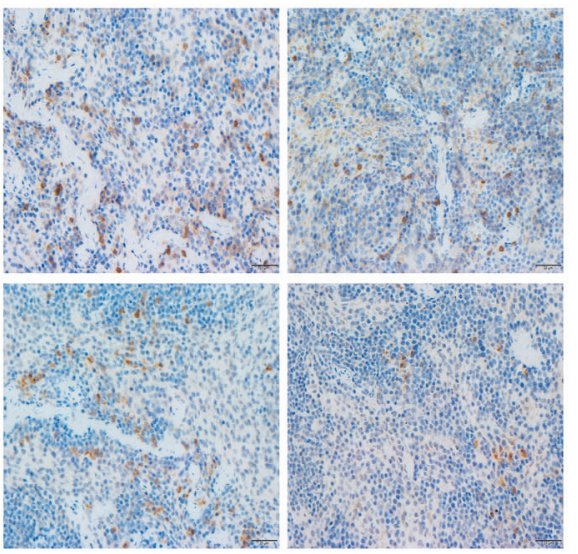

Spleen deficiency cachexia+Al
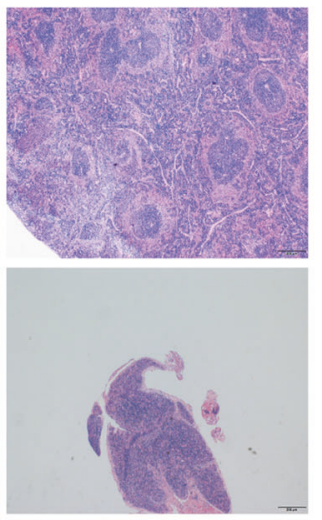

C

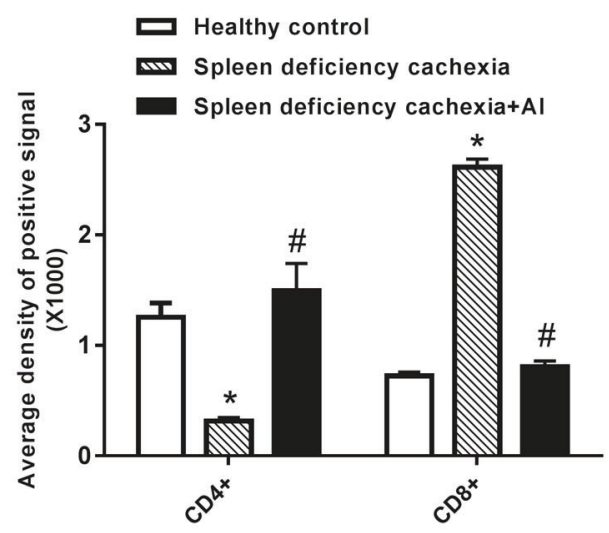

e

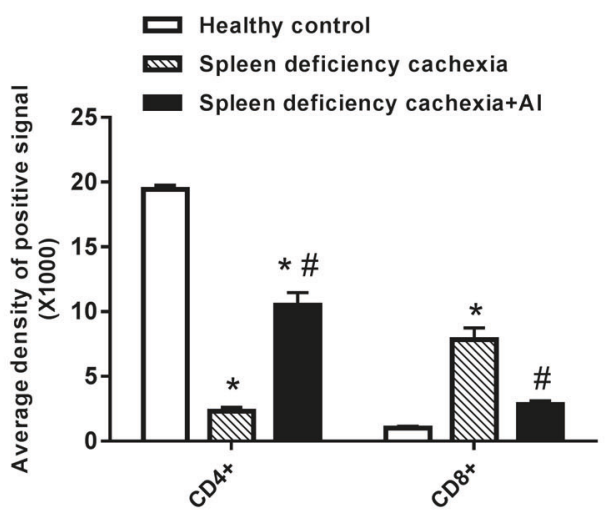

Fig. 7 Amelioration of thymus and spleen atrophy under atractylenolide I treatment. a Representative photo of H\&E-stained sections of spleen and thymus tissues. In the spleen, the red arrow indicates the red pulp, and the black arrow indicates the white pulp. In the thymus, the red arrow indicates the cortex, and the black arrow indicates the medulla. Bar $=200 \mu \mathrm{m}$. b Representative photo of immunohistochemical analysis of $\mathrm{CD}^{+}$and $\mathrm{CD} 8^{+}$cells in the spleen. Bar $=20 \mu \mathrm{m}$. c Quantitative analysis of the results from the positive signal in B. $\mathbf{d}$ Representative photo of immunohistochemistry images of $\mathrm{CD}^{+}$and $\mathrm{CD}^{+}$cells in the thymus. Bar $=20 \mu \mathrm{m}$. e Quantitative analysis of the results of the positive signal in $\mathbf{d}$. The data presented are the mean \pm SEM. ${ }^{*} P<0.05$ versus healthy control group; ${ }^{\#} P<0.05$ versus spleen-deficiency with cachexia group 
atrophy in the spleen and thymus [47]. As shown in the present study, spleen deficiency alone caused a significant decrease in both spleen weight and thymus weight. In contrast, in cancer cachexia mice inoculated with C26 cells, the weight of the thymus decreased, but the weight of the spleen increased. A decrease in thymus weight, called thymus atrophy, and an increase in spleen weight, known as splenomegaly, are frequently observed in tumor-bearing mice [48-50]. The thymus is the site of differentiation of bone marrow-derived cells committed to the T lymphocyte lineage. Cancer-related thymus atrophy might result from changes in the levels of cytokines and growth factors, and arrest of thymic differentiation in tumor-bearing mice [51]. The unique architecture of the spleen allows for interactions among the circulatory, reticuloendothelial, and immune systems [48, 52]. Therefore, splenomegaly is considered a manifestation of hyperfunction, including immune hyperplasia, extramedullary hematopoiesis, and removal of defective red blood cells, and might also reflect portal hypertension secondary to liver failure or venous obstruction and benign or malignant infiltration in diseases such as metastatic cancer [53]. The splenomegaly found in cancer cachexia mice in the present study was also observed in previous reports using mice inoculated with C26 tumor cells $[9,27]$. In patients bearing tumors, the increase in the weight of the spleen was not as significant as that in animals with xenograft tumors [54]. In particular, it has been reported that the immune function of cancer patients with spleen deficiency syndrome is markedly lower than that of normal persons [55]. Therefore, the atrophy of both the thymus and spleen in the spleen deficiency with cachexia mouse model might well mimic the characteristics of cancer patients with spleen deficiency syndrome.

Furthermore, our results suggested that the spleen deficiency with cachexia model established in the present study might be used to evaluate the anticachexia efficacy of TCMs, especially tonifying herbs. BaiZhu, Atractylodes macrocephala Koidz, is a popular TCM with "invigorating spleen" activity [56-58]. Atractylenolide I is a naturally sesquiterpene lactone and one of the main active components of BaiZhu [59]. As a tonifying TCM, Baizhu exhibited low toxicity. No toxicity was observed following intraperitoneal injection of BaiZhu extracts, even at a maximum dose of $100 \mathrm{~g} / \mathrm{kg}$, in rats [60]. The reported highest doses of atractylenolide I used in the treatment of patients or mice were $20 \mathrm{mg} / \mathrm{kg}$ [61] and $75 \mathrm{mg} / \mathrm{kg}$ [62], respectively. Atractylenolide I can be absorbed well and metabolized quickly. The main pharmacokinetic parameters, such as $T_{\max }$ and $T_{1 / 2}$ (the biological half-time), after oral administration of atractylenolide I in rats were $\sim 1-2 \mathrm{~h}$, respectively $[63,64]$. In the present study, atractylenolide I at $20 \mathrm{mg} / \mathrm{kg}$ exhibited considerable anticachexia effects against spleen deficiency with cachexia in mice. Treatment with atractylenolide I significantly ameliorated the decreases in tumor-free body weight, muscle weight, and fat weight in mice with spleen deficiency and cachexia. Mice treated with atractylenolide I exhibited improvement in the myofiber area of muscle tissue and adipocyte diameter of fat tissue. Our results also showed that atractylenolide I treatment significantly ameliorated the decrease in the levels of MHC, Myogenin, and MyoD and partly inhibited the increase in MuRF-1 in the gastrocnemius muscle tissues of mice with spleen deficiency and cachexia. MHC, myosin heavy chain protein, is the major structural protein in myotubes, and its decrease is directly related to muscle atrophy [65]. Myogenin and MyoD are both myogenic regulatory factors regulating the proliferation and differentiation of muscle cells [66] and have been identified as positive regulators of muscle size [67]. The inhibitory effects of atractylenolide I treatment on the decrease in Myogenin and MyoD might contribute to its protective effects against muscle atrophy in spleen deficiency with cachexia. Muscle-specific E3 ubiquitin ligases such as MuRF-1 are also involved in muscle atrophy in cancer cachexia [68]. Although atractylenolide I treatment could not significantly inhibit the increase in MuRF-1 in spleen deficiency with cachexia, the amelioration of MuRF-1-related protein degradation might partly contribute to its protective effects on muscle tissues.

The results of the analysis of eWAT showed that atractylenolide I treatment significantly ameliorated the decrease in the level of perilipin-1, inhibited the upregulation of HSL, and partially decreased the levels of p-HSL in mice with spleen deficiency and cachexia. Perilipin-1 belongs to the family of perilipins, whose relative expression is related to the balance of lipid storage and utilization in adipocytes [69]. HSL is an enzyme with strong diacylglycerol and cholesterol ester hydrolase activity, and weaker triacylglycerol, monoacylglycerol, and retinyl ester hydrolase activity in vitro. It is a rate-limiting enzyme that regulates adipocyte lipolysis, and its activation is dependent on phosphorylation [70]. The inhibitory effects of atractylenolide I treatment on the increase in HSL and p-HSL levels might contribute to its ability to protect fat tissues from fat lipolysis in spleen deficiency with cachexia. Furthermore, a significant increase in UCP1 was observed in eWAT from mice with spleen deficiency and cachexia. UCP1, a protein expressed exclusively in brown fat adipose tissue, is responsible for dissipating energy as heat instead of generating ATP from the oxidation of free fatty acids [71]. The upregulated expression of UCP1 in eWAT found in the present study suggested white-to-brown transdifferentiation of WAT in spleen deficiency with cachexia. Our results were in accordance with previous reports that showed a switch from white-to-brown fat and upregulated expression of UCP1 in cancer-associated cachexia $[72,73]$. Both WAT lipolysis and white-to-brown transdifferentiation of WAT could contribute to adipose tissue loss in cancer cachexia [74]. Atractylenolide I treatment partly inhibited the increase in UCP1 in eWAT from mice with spleen deficiency and cachexia. The inhibitory effects of atractylenolide I treatment on UCP1 expression might also be involved in the amelioration of adipose tissue loss in the atractylenolide I-treated group.

Treatment with atractylenolide I also ameliorated spleen atrophy and thymus atrophy in mice with spleen deficiency and cachexia. Both the weight of the spleen and the weight of the thymus were significantly higher in the spleen deficiency with cachexia $+\mathrm{Al}$ group than in the spleen deficiency with cachexia group. Treatment with atractylenolide I ameliorated the destruction of the architecture of the spleen and thymus tissues. Furthermore, the atractylenolide l-treated group showed a significant increase in the number of $\mathrm{CD}^{+}$cells and a decrease in the number of $\mathrm{CD}^{+}$cells in spleen and thymus tissues compared with those in the spleen deficiency with cachexia group. The combination of spleen deficiency and tumor inoculation induced complicated immune dysfunction in the mice. The influence of atractylenolide I on the number of $\mathrm{CD}^{+}$and $\mathrm{CD} 8^{+}$ cells in the spleen and thymus might be attributed to the amelioration of immune dysfunction in mice with spleen deficiency and cachexia, although the mechanisms require further study. Previous reports showed the immunomodulatory effects of BaiZhu, Atractylodes macrocephala Koidz [56-58]. Furthermore, atractylenolide I has been reported to exhibit antitumor activities $[59,75]$. The decrease in the number of $\mathrm{CD}^{+}$cells might be partly due to the inhibitory effects of atractylenolide I on reactions related to tumor growth, although the difference between the tumor weight of the spleen deficiency with cachexia $+\mathrm{Al}$ group and that of the spleen deficiency with cachexia group was not significant. In a randomized pilot clinical study of atractylenolide I in gastric cancer cachexia patients, atractylenolide I treatment ( $1.32 \mathrm{~g} /$ day per person for 7 weeks) ameliorated the symptoms of gastric cancer cachexia compared with the control group, which received only nutritional supplementation [76]. Notably, TCM complex prescriptions containing BaiZhu, such as SiJunZi Tang, ShenLing BaiZhu San, and ShiQuanDaBu Tang, are popularly used in China as supplementary therapy for cancer [58, 77-82]. These TCMs could improve the immune functions and nutritional status 
of cancer patients $[79,82]$. The spleen deficiency with cachexia model might be useful for screening more TCMs with anticachexia effects and studying the mechanisms of tonifying TCMs such as BaiZhu in ameliorating cancer cachexia.

Overall, we tried to establish a spleen deficiency with cancer cachexia mouse model in the present study. The aims of establishing this model are to mimic cancer cachexia patients with spleen deficiency syndrome and to screen possible anti-cachexia tonifying TCMs. The results of the present study suggested that the established spleen deficiency with cancer cachexia model exhibited both cachexia and spleen deficiency characteristics and could be used to evaluate the anti-cachexia efficacy of atractylenolide I, a TCM component. Further study is necessary in the future to clarify the pathological development of spleen deficiency with cancer cachexia and the mechanisms of anti-cachexia TCMs.

\section{ACKNOWLEDGEMENTS}

This work was supported by the National Natural Science Foundation of China (Nos. 81873056, 81872496), the Shanghai Science and Technology Innovation Action Program (No. 15140904800) and the Open Project of the State Key Laboratory of Innovative Natural Medicine, and Traditional Chinese Medicine Injections (No. QFSKL2018006).

\section{AUTHOR CONTRIBUTIONS}

WLZ and NL conducted the experiments, analyzed the data, and drafted the manuscript. QS and MF participated in the experiments. XDG revised the manuscript. $X W Z, Z Z$, and $X L$ designed the experiments, supervised the research, and revised the manuscript. All the authors reviewed and approved the manuscript.

\section{REFERENCES}

1. Argiles JM, Busquets S, Stemmler B, Lopez-Soriano FJ. Cancer cachexia: understanding the molecular basis. Nat Rev Cancer. 2014;14:754-62.

2. Fearon K, Strasser F, Anker SD, Bosaeus I, Bruera E, Fainsinger RL, et al. Definition and classification of cancer cachexia: an international consensus. Lancet Oncol. 2011;12:489-95.

3. Sadeghi M, Keshavarz-Fathi M, Baracos V, Arends J, Mahmoudi M, Rezaei N. Cancer cachexia: diagnosis, assessment, and treatment. Crit Rev Oncol/Hematol. 2018;127:91-104.

4. Mondello P, Mian M, Aloisi C, Fama F, Mondello S, Pitini V. Cancer cachexia syndrome: pathogenesis, diagnosis, and new therapeutic options. Nutr Cancer. 2015;67:12-26.

5. Baracos VE, Martin L, Korc M, Guttridge DC, Fearon KCH. Cancer-associated cachexia. Nat Rev Dis Prim. 2018;4:17105.

6. Anderson $\sqcup$, Albrecht ED, Garcia JM. Update on management of cancer-related cachexia. Curr Oncol Rep. 2017;19:3.

7. Penna F, Busquets S, Argiles JM. Experimental cancer cachexia: evolving strategies for getting closer to the human scenario. Semin Cell Dev Biol. 2016;54:20-7.

8. Giles K, Guan C, Jagoe TR, Mazurak V. Diet composition as a source of variation in experimental animal models of cancer cachexia. J Cachexia Sarcopenia Muscle. 2016;7:110-25.

9. Aulino P, Berardi E, Cardillo VM, Rizzuto E, Perniconi B, Ramina C, et al. Molecular, cellular and physiological characterization of the cancer cachexia-inducing C26 colon carcinoma in mouse. BMC Cancer. 2010;10:363.

10. Sun R, Zhang S, Lu X, Hu W, Lou N, Zhao Y, et al. Comparative molecular analysis of early and late cancer cachexia-induced muscle wasting in mouse models. Oncol Rep. 2016;36:3291-302.

11. Kumar S, Kishimoto H, Chua HL, Badve S, Miller KD, Bigsby RM, et al. Interleukin-1 alpha promotes tumor growth and cachexia in MCF-7 xenograft model of breast cancer. Am J Pathol. 2003;163:2531-41.

12. Pin F, Barreto R, Kitase $Y$, Mitra S, Erne CE, Novinger LJ, et al. Growth of ovarian cancer xenografts causes loss of muscle and bone mass: a new model for the study of cancer cachexia. J Cachexia Sarcopenia Muscle. 2018;9:685-700.

13. Terawaki K, Kashiwase $Y$, Sawada Y, Hashimoto H, Yoshimura M, Ohbuchi K, et al. Development of ghrelin resistance in a cancer cachexia rat model using human gastric cancer-derived 85 As 2 cells and the palliative effects of the Kampo medicine rikkunshito on the model. PLoS ONE. 2017;12:e0173113.
14. Musolino V, Palus S, Latouche C, Gliozzi M, Bosco F, Scarano F, et al. Cardiac expression of neutrophil gelatinase-associated lipocalin in a model of cancer cachexia-induced cardiomyopathy. ESC Heart Fail. 2019;6:89-97.

15. Ballaro R, Costelli P, Penna F. Animal models for cancer cachexia. Curr Opin Support Palliat Care. 2016;10:281-7.

16. Ji $\mathrm{Q}$, Luo YQ, Wang WH, Liu X, Li $Q$, Su SB. Research advances in traditional Chinese medicine syndromes in cancer patients. J Integr Med. 2016;14:12-21.

17. Xu L, Lu JW. Experience for treating CC in TCM. Chin J Clin Res. 2012;4:2.

18. Ma T. Study of cancer-related anorexia cachexia syndrome from spleen deficiency treatment. Inf Tradit Chin Med. 2009;26:3.

19. Li P, Xin B, Qian WW. Progress in treatment of cancer anorexia using traditional Chinese medicine in recent 6 years. Mod Tradit Chin Med. 2017;37:3.

20. Xi L, Zhu J, Zhang H, Muktiali M, Xu C, Wu A. Epidemiological trends in gastrointestinal cancers in China: an ecological study. Dig Dis Sci. 2019;64:532-43.

21. Schwarz S, Prokopchuk O, Esefeld K, Groschel S, Bachmann J, Lorenzen S, et al. The clinical picture of cachexia: a mosaic of different parameters (experience of 503 patients). BMC cancer. 2017;17:130.

22. Ozorio GA, Barao K, Forones NM. Cachexia stage, patient-generated subjective global assessment, phase angle, and handgrip strength in patients with gastrointestinal cancer. Nutr Cancer. 2017;69:772-79.

23. Sun DZ, Liu L, Jiao JP, Wei PK, Jiang LD, Xu L. Syndrome characteristics of traditional Chinese medicine: summary of a clinical survey in 767 patients with gastric cancer. J Chin Integr Med. 2010;8:332-40.

24. Sun XG, Lin XC, Diao JX, Yu ZL, Li K. Pi (Spleen)-deficiency syndrome in tumor microenvironment is the pivotal pathogenesis of colorectal cancer immune escape. Chin J Integr Med. 2016;22:789-94.

25. Bindels LB, Neyrinck AM, Loumaye A, Catry E, Walgrave H, Cherbuy C, et al Increased gut permeability in cancer cachexia: mechanisms and clinical relevance. Oncotarget. 2018;9:18224-38.

26. Molinari F, Pin F, Gorini S, Chiandotto S, Pontecorvo L, Penna F, et al. The mitochondrial metabolic reprogramming agent trimetazidine as an 'exercise mimetic' in cachectic C26-bearing mice. J Cachexia Sarcopenia Muscle. 2017;8:954-73.

27. Bonetto A, Rupert JE, Barreto R, Zimmers TA. The colon-26 carcinoma tumorbearing mouse as a model for the study of cancer cachexia. J Vis Exp. 2016;117: e54893. https://doi.org/10.3791/54893.

28. Shum AMY, Poljak A, Bentley NL, Turner N, Tan TC, Polly P. Proteomic profiling of skeletal and cardiac muscle in cancer cachexia: alterations in sarcomeric and mitochondrial protein expression. Oncotarget. 2018;9:22001-22.

29. Jin J, Miao C, Wang Z, Zhang W, Zhang X, Xie X, et al. Design and synthesis of aryloxypropanolamine as beta3-adrenergic receptor antagonist in cancer and lipolysis. Eur J Med Chem. 2018;150:757-70.

30. Yoshimura M, Shiomi Y, Ohira Y, Takei M, Tanaka T. Z-505 hydrochloride, an orally active ghrelin agonist, attenuates the progression of cancer cachexia via anabolic hormones in Colon 26 tumor-bearing mice. Eur J Pharmacol. 2017:811:30-7.

31. Villars FO, Pietra C, Giuliano C, Lutz TA, Riediger T. Oral treatment with the ghrelin receptor agonist HM01 attenuates cachexia in mice bearing colon-26 (C26) tumors. Int J Mol Sci. 2017;18:E986.

32. Guo D, Wang C, Wang Q, Qiao Z, Tang H. Pantoprazole blocks the JAK2/STAT3 pathway to alleviate skeletal muscle wasting in cancer cachexia by inhibiting inflammatory response. Oncotarget. 2017;8:39640-48.

33. Hang W, Zhang HY, Huang SP. The study of composite factors make spleendeficient models in rats. Lishizhen Med Mater Medica Res. 2015;26:3.

34. Wu TS, Zhang HY, Zhang Z, Yang GL. Evaluation of methods in establishing spleen-deficiency animal models. J Tradit Chin Med. 2015;56:6.

35. Meng JY, Ying SL, Ma Y, Gao JL, Xu DQ. Experimental study of spleen-deficiency animal model. Tianjin Tradit Chin Med. 1996;6:3.

36. Wang H, Lai YJ, Chan YL, Li TL, Wu CJ. Epigallocatechin-3-gallate effectively attenuates skeletal muscle atrophy caused by cancer cachexia. Cancer Lett. 2011;305:40-9.

37. Choi YK, Jung KY, Woo SM, Yun YJ, Jun CY, Park JH, et al. Effect of Sipjeondaebotang on cancer-induced anorexia and cachexia in CT-26 tumor-bearing mice. Mediat Inflamm. 2014;2014:736563.

38. Kim A, Im M, Ma JY. Sosihotang ameliorates cachexiarelated symptoms in mice bearing colon 26 adenocarcinoma by reducing systemic inflammation and muscle loss. Oncol Rep. 2016;35:1841-50.

39. Ming-Hua C, Bao-Hua Z, Lei Y. Mechanisms of anorexia cancer cachexia syndrome and potential benefits of traditional medicine and natural herbs. Curr Pharm Biotechnol. 2016;17:1147-52.

40. Zhuang $P$, Zhang J, Wang $Y$, Zhang $M$, Song L, Lu Z, et al. Reversal of muscle atrophy by Zhimu and Huangbai herb pair via activation of IGF-1/Akt and autophagy signal in cancer cachexia. Support Care Cancer 2016;24:1189-98. 
41. Chen YY, Chen NJ, Wu DH, Lan YQ. Clinical observation of treating advanced cancer cachexia patients with Sen-Ling-Bai-Zhu-San. Fujian J Tradit Chin Med. 2008;39:2.

42. Xu L, Zhang XJ, Yang GL. Influence of sijunzi decoction on serum cytokines of mice with cancer cachexia. Chin Arch Tradit Chin Med. 2015;33:907.

43. Cai HB, Luo RW, Yang CB, Pan CH, Zuo Q, Wang YG. Observation of clinical efficacy of Bu-Zhong-Yi-Qi decotion in treatment of cancer anorexia cachixia syndromes. New Tradit Chin Med. 2003;35:2.

44. Miao C, Lv Y, Zhang W, Chai X, Feng L, Fang Y, et al. Pyrrolidine dithiocarbamate (PDTC) attenuates cancer cachexia by affecting muscle atrophy and fat lipolysis. Front Pharmacol. 2017:8:915.

45. Liu W, Lu Y, Chai X, Liu X, Zhu T, Wu X, et al. Antitumor activity of TY-011 against gastric cancer by inhibiting Aurora A, Aurora B, and VEGFR2 kinases. J Exp Clin Cancer Res. 2016;35:183.

46. Chen Z, Wang P. Clinical distribution and molecular basis of traditional Chinese medicine ZHENG in cancer. Evid Based Complement Altern Med. 2012;2012:783923.

47. Zhao X, Chen T, Meng F, Wang H, Tian P, Tang X, et al. Therapeutic effect of herb residue fermentation supernatant on spleendeficient mice. Mol Med Rep. 2018;17:2764-70.

48. Fang JJ, Zhu ZY, Dong H, Zheng GQ, Teng AG, Liu AJ. Effect of spleen lymphocytes on the splenomegaly in hepatocellular carcinoma-bearing mice. Biomed Environ Sci. 2014;27:17-26.

49. Kaiserlian D, Savino W, Hassid J, Dardenne M. Studies of the thymus in mice bearing the Lewis lung carcinoma. III. Possible mechanisms of tumor-induced thymic atrophy. Clin Immunol Immunopathol. 1984;32:316-25.

50. Hodgson A, Wier EM, Fu K, Sun X, Wan F. Ultrasound imaging of splenomegaly as a proxy to monitor colon tumor development in $\mathrm{Apc}(\min 716 /+)$ mice. Cancer Med. 2016;5:2469-76.

51. Carrio R, Lopez DM. Insights into thymic involution in tumor-bearing mice. Immunol Res. 2013:57:106-14.

52. Chadburn A. The spleen: anatomy and anatomical function. Semin Hematol. 2000;37(1Suppl 1):13-21.

53. Zimmers TA, Fishel ML, Bonetto A. STAT3 in the systemic inflammation of cancer cachexia. Semin Cell Dev Biol. 2016:54:28-41.

54. Heymsfield SB, McManus CB. Tissue components of weight loss in cancer patients. A new method of study and preliminary observations. Cancer. 1985;55(1 Suppl):238-49.

55. Yu RC, Guan CF, Zhang JH. Immune function of cancer patients with spleendeficiency syndrome. Chin J Mod Dev Tradit Med. 1990;10:535-7, 16.

56. Xu S, Qi X, Liu Y, Liu Y, Lv X, Sun J, et al. UPLC-MS/MS of Atractylenolide I, Atractylenolide II, Atractylenolide III, and Atractyloside A in rat plasma after oral administration of raw and wheat bran-processed atractylodis rhizoma. Molecules. 2018;23:E3234. pii

57. Kwak TK, Jang HS, Lee MG, Jung YS, Kim DO, Kim YB, et al. Effect of orally administered Atractylodes macrocephala Koidz water extract on Macrophage and T Cell inflammatory response in mice. Evid Based Complement Alternat Med. 2018;2018:4041873.

58. Zee-Cheng RK. Shi-quan-da-bu-tang (ten significant tonic decoction), SQT. A potent Chinese biological response modifier in cancer immunotherapy, potentiation and detoxification of anticancer drugs. Methods Find Exp Clin Pharmacol. 1992;14:725-36.

59. Long $F$, Wang $T$, Jia $P$, Wang $H$, Qing $Y$, Xiong $T$, et al. Anti-tumor effects of atractylenolide-I on human ovarian cancer cells. Med Sci Monit. 2017;23:571-9.

60. Zhu B, Zhang QL, Hua JW, Cheng WL, Qin LP. The traditional uses, phytochemistry, and pharmacology of Atractylodes macrocephala Koidz.: A review. J Ethnopharmacol. 2018;226:143-67.

61. Liu Y, Ye F, Qiu GQ, Zhang M, Wang R, He QY, et al. Effects of lactone I from Atractylodes macrocephala Koidz on cytokines and proteolysis-inducing factors in cachectic cancer patients. Acad J First Med Coll PLA. 2005;25:1308-11.

62. Xiao Q, Zheng F, Tang Q, Wu JJ, Xie J, Huang HD, et al. Repression of PDK1- and LncRNA HOTAIR-mediated EZH2 gene expression contributes to the enhancement of atractylenolide 1 and erlotinib in the inhibition of human lung cancer cells. Cell Physiol Biochem. 2018;49:1615-32.

63. Li Y, Zhang Y, Wang Z, Zhu J, Tian Y, Chen B. Quantitative analysis of atractylenolide I in rat plasma by LC-MS/MS method and its application to pharmacokinetic study. J Pharm Biomed Anal. 2012;58:172-6.

64. Zhu ZM, Li HC, Luo JB. Simultaneous determination of atractylenolide I, II, III by HPLC-MS method and its application to pharmacokinetic study. Pharmacol Clin Chin Mater Medica. 2013;29:5.

65. Johns N, Stephens NA, Fearon KC. Muscle wasting in cancer. Int J Biochem Cell Biol. 2013;45:2215-29.

66. Hernandez-Hernandez JM, Garcia-Gonzalez EG, Brun CE, Rudnicki MA. The myogenic regulatory factors, determinants of muscle development, cell identity and regeneration. Semin Cell Dev Biol. 2017;72:10-8.

67. Stewart CE, Rittweger J. Adaptive processes in skeletal muscle: molecular regulators and genetic influences. J Musculoskelet Neuronal Interact. 2006;6:73-86.

68. Yuan L, Han J, Meng Q, Xi Q, Zhuang Q, Jiang Y, et al. Muscle-specific E3 ubiquitin ligases are involved in muscle atrophy of cancer cachexia: an in vitro and in vivo study. Oncol Rep. 2015;33:2261-8.

69. Sztalryd C, Brasaemle DL. The perilipin family of lipid droplet proteins: gatekeepers of intracellular lipolysis. Biochim Biophys Acta Mol Cell Biol Lipids. 2017;1862(10 Pt B):1221-32.

70. Anthonsen MW, Ronnstrand L, Wernstedt C, Degerman E, Holm C. Identification of novel phosphorylation sites in hormone-sensitive lipase that are phosphorylated in response to isoproterenol and govern activation properties in vitro. J Biol Chem. 1998;273:215-21.

71. Klaus S, Casteilla L, Bouillaud F, Ricquier D. The uncoupling protein UCP: a membraneous mitochondrial ion carrier exclusively expressed in brown adipose tissue. Int J Biochem. 1991;23:791-801.

72. Petruzzelli M, Schweiger M, Schreiber R, Campos-Olivas R, Tsoli M, Allen J, et al. A switch from white to brown fat increases energy expenditure in cancerassociated cachexia. Cell Metab. 2014;20:433-47.

73. Bing C, Brown M, King P, Collins P, Tisdale MJ, Williams G. Increased gene expression of brown fat uncoupling protein (UCP)1 and skeletal muscle UCP2 and UCP3 in MAC16-induced cancer cachexia. Cancer Res. 2000;60:2405-10.

74. Dalal S. Lipid metabolism in cancer cachexia. Ann Palliat Med. 2019;8:13-23.

75. Fu XQ, Chou JY, Li T, Zhu PL, Li JK, Yin CL, et al. The JAK2/STAT3 pathway is involved in the anti-melanoma effects of atractylenolide I. Exp Dermatol. 2018;27:201-4.

76. Liu Y, Jia Z, Dong L, Wang R, Qiu G. A randomized pilot study of atractylenolide I on gastric cancer cachexia patients. Evid Bbased Complement Altern Med. 2008;5:337-44

77. Lin X, Xu W, Shao M, Fan Q, Wen G, Li C, et al. Shenling Baizhu San supresses colitis associated colorectal cancer through inhibition of epithelial-mesenchymal transition and myeloid-derived suppressor infiltration. BMC Complement Altern Med. 2015;15:126.

78. Zhang B, Huang G, Zhang $Y$, Chen X, Hu P, Xu B. Clinical observation on prevention of "jia wei si jun zi tang" from damage of hepatic reserving function after intervention of liver cancer. J Chin Med Mater. 2004;27:387-9.

79. Cai J, Wang H, Zhou S, Wu B, Song HR, Xuan ZR. Effect of Sijunzi Decoction and enteral nutrition on T-cell subsets and nutritional status in patients with gastric cancer after operation: a randomized controlled trial. J Chin Integr Med. 2008;6:37-40.

80. Cheon C, Kang S, Ko Y, Kim M, Jang BH, Shin YC, et al. Sipjeondaebo-tang in patients with breast cancer with fatigue: a protocol for a pilot, randomised, double-blind, placebo-controlled, cross-over trial. BMJ Open. 2018;8:e021242.

81. Wang $\mathrm{CY}$, Bai $\mathrm{XY}$, Wang $\mathrm{CH}$. Traditional Chinese medicine: a treasured natural resource of anticancer drug research and development. Am J Chin Med. 2014;42:543-59.

82. Cheon C, Yoo JE, Yoo HS, Cho CK, Kang S, Kim M, et al. Efficacy and safety of sipjeondaebo-tang for anorexia in patients with cancer: a pilot, randomized, double-blind, placebo-controlled trial. Evid Based Complement Altern Med. 2017:2017:8780325. 